\title{
A Hierarchical Inertial Control Scheme for Multiple Wind Farms with BESSs Based on ADMM
}

Bao, Weiyu; Wu, Qiuwei; Ding, Lei; Huang, Sheng; Terzija, Vladimir

Published in:

IEEE Transactions on Sustainable Energy

Link to article, DOI:

10.1109/TSTE.2020.2995101

Publication date:

2021

Document Version

Peer reviewed version

Link back to DTU Orbit

Citation (APA):

Bao, W., Wu, Q., Ding, L., Huang, S., \& Terzija, V. (2021). A Hierarchical Inertial Control Scheme for Multiple Wind Farms with BESSs Based on ADMM. IEEE Transactions on Sustainable Energy, 12(2), 751 - 760. https://doi.org/10.1109/TSTE.2020.2995101

\section{General rights}

Copyright and moral rights for the publications made accessible in the public portal are retained by the authors and/or other copyright owners and it is a condition of accessing publications that users recognise and abide by the legal requirements associated with these rights.

- Users may download and print one copy of any publication from the public portal for the purpose of private study or research.

- You may not further distribute the material or use it for any profit-making activity or commercial gain

- You may freely distribute the URL identifying the publication in the public portal 


\title{
A Hierarchical Inertial Control Scheme for Multiple Wind Farms with BESSs Based on ADMM
}

\author{
Weiyu Bao, Student Member, IEEE, Qiuwei Wu, Senior Member, IEEE, Lei Ding, Member, IEEE, \\ Sheng Huang, Vladimir Terzija, Fellow, IEEE
}

\begin{abstract}
Wind farms (WFs) can provide inertial response to power systems through synthetic inertial control. In large scale power systems with high penetration level of wind power, WFs are located in different areas with different wind speed conditions, and it is necessary to coordinate them during inertial control. This paper proposes a hierarchical inertial control (HIC) scheme consisting of a WF level coordination and a wind turbine (WT)/battery energy storage system (BESS) level coordination in active power outputs during inertial control process, in order to improve the system frequency response and reduce the operational cost of each WF. First, the optimal active power references of multiple WFs during inertial control are determined to improve the frequency nadir of the system. Second, the active power references of the WTs/BESS of each WF is optimized to avoid over-deceleration of the WTs' rotor, and minimize the wind energy loss and degradation cost of the BESS. The optimization is achieved by developing a model predictive control (MPC) problem and solving it using the alternating direction method of multipliers (ADMM) to improve the computation efficiency. Simulations on 5 WFs integrated into IEEE 39-bus system were conducted to validate the proposed HIC scheme.
\end{abstract}

Index Terms-hierarchical control, inertial control, wind farm (WF), system frequency response

\section{INTRODUCTION}

W IND power is regarded as one of the most promising renewable energy resources (RESs) and it has been growing significantly in recent years. With large scale integration of wind power into the power system, conventional fossil fuel-based power plants are being gradually replaced by wind farms (WFs). Since the rotational speed of the inverter-based wind turbines (WTs) is decoupled from the system frequency, WTs do not inherently contribute to system inertia, which is mostly provided by conventional power plants. Therefore, high penetration of wind power results in a low-inertia power system, and introduces more challenges to the system frequency stability [1]. To cope with the problem of decreasing system

Corresponding author: Qiuwei Wu, (e-mail: qw@elektro.dtu.dk), Lei Ding, (e-mail: dinglei@sdu.edu.cn).

W. Bao and L. Ding are with the Key Laboratory of Power System Intelligent Dispatch and Control of Ministry of Education, School of Electrical Engineering, Shandong University, Jinan 250061, China (email: bwysdu@163.com; dinglei@sdu.edu.cn).

Q. Wu and S. Huang are with the Center for Electric Power and Energy (CEE), Department of Electrical Engineering, Technical University of Denmark (DTU), Kgs. Lyngby 2800, Denmark (e-mail: qw@elektro.dtu.dk; huang98123@163.com).

V. Terzija is with School of Electrical \& Electronic Engineering, University of Manchester, Manchester M13 9PL, U.K. (e-mail: Vladimir.Terzija@manchester.ac.uk).

This work was supported by the National Key R\&D Program of China (2018YFB0904004). inertia, it is necessary for WFs to provide inertial response during frequency disturbances.

In order to provide inertial response, inertial control of WTs was proposed [2], and a large number of studies have been conducted to investigate its influence on system dynamics and improve its control performance [3]- [9]. The basic concept of inertial control is to increase the active power output of the WT temporarily and release the kinetic energy stored in the rotating mass of the WT after frequency disturbances, in order to support the system frequency recovery. Owing to the instant response of active power control of the WTs and the considerable available kinetic energy stored in the WT's rotating mass, inertial control can significantly improve the system frequency stability by reducing the rate of change of frequency (RoCoF) and mitigating frequency deviations after disturbances. In [3], the effects of inertial control with different control parameters on the frequency response of autonomous island systems were investigated. It was concluded that enhancing the inertial response of WTs has a significant positive effect on the system operation. To improve control performance, variable gains for inertial control has been studied in the literature. In [4] and [5], the gains of inertia control were set to be proportional to the WT's releasable kinetic energy in order to ensure stable operation of the WT. Moreover, inertial control with a variable gain depending on the RoCoF was proposed in [6] and [7] and it can improve the system frequency nadir after the disturbance. As another form of inertial control, stepwise inertial control rapidly increases the active power output of the WT by a constant value when detecting an event. Stepwise inertial control was studied in [8] and [9] by proposing incremental power references varying with WT's rotor speed and wind power penetration level. The proposed approach provides a promising solution for the inertial control of WTs in a power system with high wind power penetration.

Despite its contribution to the system frequency stability, the application of the inertial control also raises some problems.

1. Over-deceleration of the rotor speed. After the inertial control is activated, the rotor speed of the WT will decrease as a result of releasing kinetic energy to the power system. If the rotor speed decelerates to its minimum limit, the inertial control of the WT will be terminated by decreasing its active power output to restore the rotor speed. This termination will cause a severe secondary frequency drop [10] to the power system. In this regard, it is necessary to prevent the over-deceleration of the rotor speed of the WTs, in order to guarantee that the inertial control will not be terminated 
too early during the system frequency recovery. Accordingly, the secondary frequency drop caused by the termination of the inertial control can be reduced. Since the dynamic of the rotor speed is closely associated with the active power outputs of the WTs, studies have been conducted to prevent the over-deceleration of the rotor speed by modifying the active power control loop of the WTs or introducing new methods to control active power outputs of the WTs during inertial control. For example, the concept of "active rotor speed protection" was proposed in [11] to adjust the active power outputs of the WTs dynamically during the inertial control to mitigate the deceleration of the WTs' rotor speed. An extended state observer (ESO)-based inertial control for WTs was proposed in [12] to prevent the rotor speed from an extensive decrease. For the same purpose, optimization-based inertial control schemes [13], [14] or intelligent learning-based inertial control system for WTs [15] were designed.

2. Increasing operation cost of the WF. During the inertial control process, due to the deceleration of the rotor, the WTs have to deviate from the maximum power point tracking (MPPT) operating point, which means that the captured wind energy will be reduced. The loss of captured wind energy would not be a problem when the capacity of the WF is low. However, for a large-scale WF with high capacity, the annual wind energy loss caused by inertial control is not negligible and it can raise the operation cost of the WF. Previous studies have shown that the loss of captured wind energy can be mitigated by adopting variable control parameters according to wind speed [16], [17] or optimization algorithms [18], [19]. However, the above approaches may also have a negative effect on the system frequency recovery.

In recent years, battery energy storage systems (BESSs) have been widely applied in WFs due to flexible charging and discharging characteristics [20], and BESS can help improve the performance of inertial control [10], [21], [22]. However, due to the high cost associated with the investment and maintenance of the BESS, participation of BESS to inertial control may also lead to an increasing operation cost of the WF.

Therefore, considering the wind energy loss and degradation cost of the BESS, the increasing operation cost of the WF during inertial control still remains as a challenging problem.

3. lack of coordination among multiple WFs. In the existing studies, most of the inertial control strategies are centralized control which only focuses on WTs providing inertial response independently based on local frequency measured at the point of common coupling (PCC) of the WF. However, at the onset of the frequency event, the RoCoF might be different at different buses in the power system. Hence, a locally implemented centralized inertial control strategy might not yield an optimal result [23]. On the other hand, in power systems with a high penetration level of wind power, with the growth of the number and scale of the WFs, WFs may cover extended areas of hundreds of square miles, and the WFs will be located in different areas with different wind speed conditions [24]. If the WFs provide inertial response after detecting frequency disturbances without any coordination, it is possible that they release excessive or insufficient kinetic energy to the power system. It results in worse system frequency excursions. Therefore, coordination among WFs during inertial control is necessary to improve system frequency response and realize the optimal operation of WFs. A few recent studies have proposed coordination schemes of WTs in a single WF during inertial control [18], [25]- [27]. However, it is challenging to extend these schemes to coordinate multiple WFs because of the computation burden and communication delay. To the best of the authors' knowledge, how to coordinate multiple WFs in a power system during inertial control has not been studied. Therefore, a comprehensive inertial control scheme focusing on coordination of both WFs and WTs is required.

Therefore, this paper proposes a hierarchical inertial control (HIC) scheme for multiple WFs consisting of BESSs. The HIC scheme includes a WF level coordination and a WT/BESS level coordination in active power outputs during the inertial control process. The WF level coordination aims to improve the system frequency nadirs after disturbances, and it is achieved by optimally determining different active power references for the WFs considering their operating conditions. The WT/BESS level coordination is to mitigate the over-deceleration of the WTs' rotor speed and reduce the operational cost of the WF, which is realized by formulating a model predictive control (MPC)-based optimization problem and solving it in a distributed manner using the Alternating Direction Method of Multipliers (ADMM) algorithm. The main contributions of this paper can be summarized as follows:

- A hierarchical inertial control structure is designed to coordinate active power outputs of multiple WFs and WTs/BESSs at two levels with different objectives after disturbances.

- Based on the prediction of the system frequency nadirs after disturbances, two linear programming (LP) problems are formulated to optimally determine the active power outputs of the WFs and improve the frequency nadirs.

- An MPC-based optimization problem is formulated to determine the active power outputs of the WTs/BESS with the objective of mitigating the over-deceleration of the WTs' rotor speed and minimizing the operation cost of the WF. ADMM algorithm is used to solve the optimization problem to improve the computation efficiency.

The rest of the paper is organized as follows. In Section II, the control structure of the HIC scheme is presented. In Section III, the coordination for WFs is proposed. In Section $\mathrm{IV}$, an MPC-based optimization problem is formulated to achieve the coordination of the WTs/BESS and it is solved using the ADMM-based distributed solution method. The simulation results are presented in Section V, followed by the conclusions.

\section{Control Architecture}

The structure of the proposed HIC scheme is illustrated in Fig. 1. For the WF level coordination, after detecting frequency disturbance, the initial RoCoF is measured by the WFs at the PCCs and then sent to a central agent named "WFC Operator". At the same time, the operating conditions of the WFs are also 


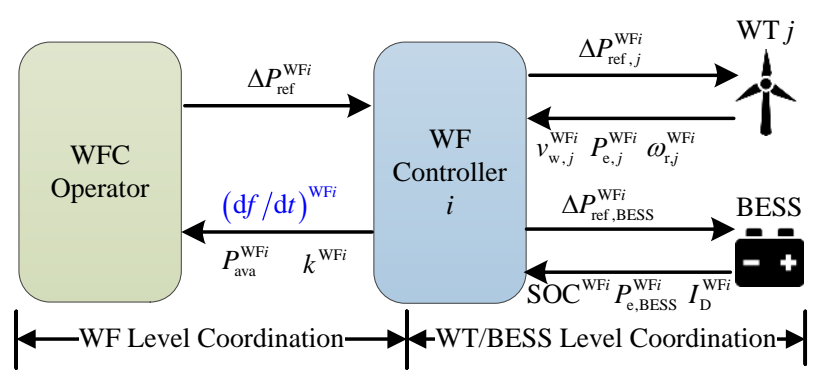

Fig. 1. Overview of HIC scheme.

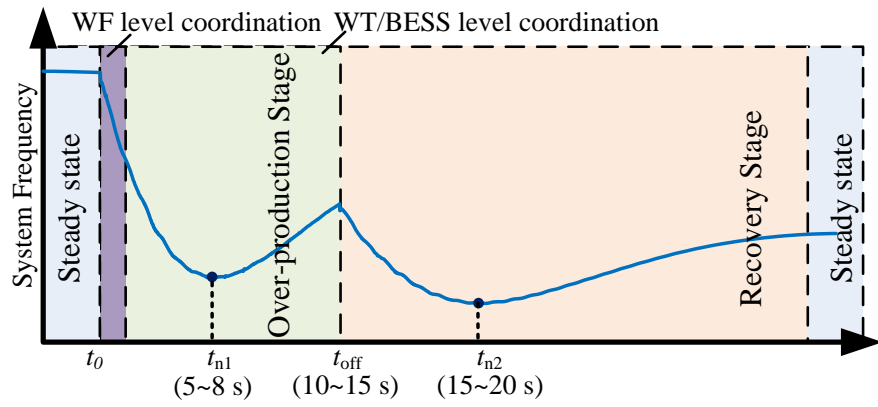

Fig. 2. System frequency response with HIC scheme.

sent to the WFC Operator. After receiving the information from the WFs, the WFC Operator estimates the magnitude of the disturbance, and then predicts the system frequency nadirs during inertial control process with the estimated disturbance magnitude. Afterwards, based on the prediction of the system frequency nadirs, the references for the active power outputs of the WFs can be optimally determined by solving two LP problems with the objective of minimizing the frequency excursions at the nadirs. The LP problems can be solved within several milliseconds. The active power reference of the $i$ th WF is denoted as $\Delta P_{\mathrm{ref}}^{\mathrm{WF} i}$.

For the WT/BESS level coordination, in the $i$ th WF, after receiving the $\Delta P_{\text {ref }}^{\mathrm{WF} i}$ from the WFC Operator, the active power references of the WTs and the BESS can be determined and then sent to the WTs/BESS by the central agent of the WF, which is named "WF controller". This determination is achieved by solving an MPC-based optimization problem during each control period. The objective is to minimize over-deceleration of the WTs' rotor speed, wind energy loss and degradation cost of the BESS. Meanwhile, the $\Delta P_{\text {ref }}^{\mathrm{WF} i}$ received from the WFC Operator is satisfied as an equality constraint of the optimization problem. To reduce the computation burden of the WF controller, this optimization problem is solved in a distributed manner using the ADMM algorithm. For each control period, the WF controller solves an unconstrained problem and each local agent at the WT/BESS solves a local small-scale constrained problem based on the WT/BESS's local operating condition and the iteration information from the WF controller. The total computation time of the WF and WT/BESS controllers is less than the control period so that the coordination is suitable for the online calculation. The active power reference of the $j$ th WT in the

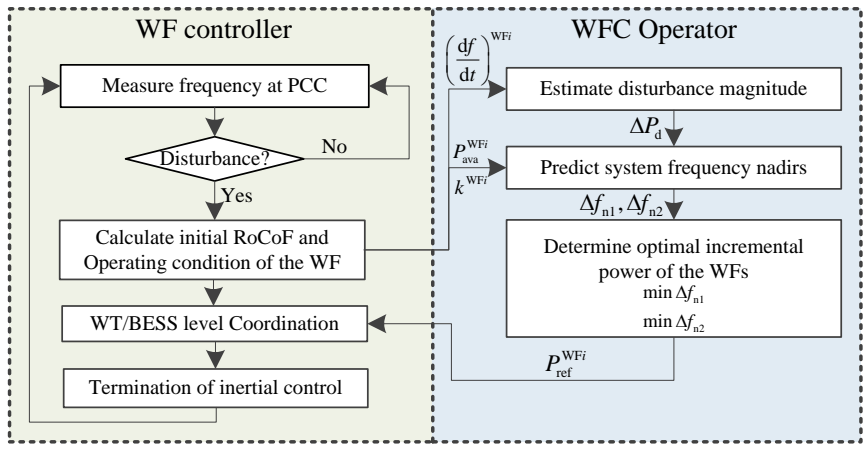

Fig. 3. Flowchart of WF level active power output coordination.

$i$ th WF is denoted as $\Delta P_{\mathrm{ref}, j}^{\mathrm{WF} i}$, and the active power reference of the BESS in the the $i$ th WF is denoted as $\Delta P_{\text {ref.BESS }}^{\mathrm{WF} i}$.

With the proposed HIC scheme, the system frequency response after a disturbance is shown in Fig. 2. Before the disturbance, the system frequency is in the steady state corresponding to the nominal frequency value (i.e., $60 \mathrm{~Hz}$ in the U.S. power system). At time $t_{0}$, a disturbance happens and causes a decline of the system frequency. In a short time period, the WF level coordination is implemented. Then the inertial control of the WTs is activated by entering the overproduction stage, in which the WT/BESS level coordination is achieved by coordinating the active power outputs of the WTs/BESS. The system frequency nadir is at time $t_{\mathrm{n} 1}$ and then the frequency starts increasing. At time $t_{\text {off }}$, the inertial control is terminated, and the WTs enters the recovery stage by decreasing the active power output to recover the rotor speed, which causes another system frequency drop. After the recovery stage, the WTs' operating points return to the MPPT points and the system frequency reaches a new steady state.

\section{WF LeVel Active Power OUtPut CoORdination}

The WF level coordination for active power outputs aims to improve the frequency nadir after the system disturbance. Fig. 3 shows the flowchart of the WF level active power output coordination. After detecting the frequency disturbance, each WF controller sends the initial RoCoF and the operating condition of the WF to the WFC Operator. Then the WFC Operator estimates the magnitude of the disturbance and predicts the system frequency nadirs. The optimal incremental power of the WFs will then be determined and sent to each WF controller. Afterwards, the inertial control of each WF will be activated and the WT/BESS level coordination will be implemented. Details of the WF level active power output coordination are described as follows.

\section{A. Estimation of Disturbance Magnitude}

After the WFs detect frequency excursions at their PCCs, the initial system RoCoF, which is necessary for estimating the disturbance magnitude, should be estimated first.

For the $i$ th WF, the initial RoCoF at the PCC $(\mathrm{d} f / \mathrm{d} t)^{\mathrm{WF} i}$ can be approximated as,

$$
\left(\frac{\mathrm{d} f}{\mathrm{~d} t}\right)^{\mathrm{WF} i}=\left(\frac{\Delta f}{\Delta t}\right)^{\mathrm{WF} i}, \quad \forall i \in \mathcal{N}_{\mathrm{F}}
$$


where $\Delta f$ is the frequency deviation measured at the PCC of the WF at the period of time $\Delta t$ after the disturbance, and $\mathcal{N}_{\mathrm{F}}$ is the number of WFs. It was mentioned in [23] that a larger $\Delta t$ contributes to more accurate estimation of the system RoCoF. On the other hand, a larger $\Delta t$ also leads to more delayed activation of the inertial control. In this paper, $\Delta t$ is set as $300 \mathrm{~ms}$ in order to strike a balance between the accuracy of system RoCoF estimation and the speed of the inertial control activation.

The initial system RoCoF can be estimated as the average value of the initial RoCoF at the PCCs the WFs, i.e.,

$$
\frac{\mathrm{d} f}{\mathrm{~d} t}=\sum_{i=1}^{\mathcal{N}_{\mathrm{F}}}\left(\frac{\mathrm{d} f}{\mathrm{~d} t}\right)^{\mathrm{WF} i} \frac{1}{\mathcal{N}_{\mathrm{F}}}
$$

According to the swing equation, when damping is neglected, the active power imbalance caused by the disturbance, i.e., the magnitude of the disturbance, can be calculated as [28],

$$
\Delta P_{\mathrm{d}}=\frac{H_{\mathrm{s}}}{f_{\mathrm{b}}} \frac{\mathrm{d} f}{\mathrm{~d} t}
$$

where $H_{\mathrm{s}}$ is the system inertia constant and $f_{\mathrm{b}}$ is the nominal frequency of the power system.

\section{B. Operating Conditions of WFs}

As shown in Fig. 2, the process of inertial control includes two stages: 1) over-production stage, in which the WF injects a certain amount of incremental active power (denoted as $\Delta P_{\mathrm{ref}}^{\mathrm{WF} i}$ ) to the system and supports the system frequency recovery, 2) recovery stage, in which the active power outputs of the WTs in the WF decrease to the MPPT points, in order to recover the rotor speed of the WTs. The time when the over-production stage ends is named termination time and denoted as $t_{\text {off }}$. The power decrease of the $i$ th WF at $t_{\text {off }}$ is denoted as $\Delta P_{\mathrm{off}}^{\mathrm{WF} i}$. A larger $\Delta P_{\mathrm{ref}}^{\mathrm{WF} i}$ means that the WF can provide more support to system frequency recovery during over-production stage. However, since the active power outputs of the WTs return back to the MPPT points during recovery stage, a larger $\Delta P_{\mathrm{ref}}^{\mathrm{WF} i}$ may also lead to a larger $\Delta P_{\mathrm{off}}^{\mathrm{WF} i}$, which causes a severer secondary frequency drop. The relation between $\Delta P_{\mathrm{off}}^{\mathrm{WF} i}$ and $\Delta P_{\mathrm{ref}}^{\mathrm{WF} i}$ of the $\mathrm{WF}$ is dependent on the operating condition of the WF. Therefore, in order to improve the overall system frequency response after the disturbance, it is necessary for each WF to calculate its available incremental power and the relation between $\Delta P_{\mathrm{off}}^{\mathrm{WF} i}$ and $\Delta P_{\mathrm{ref}}^{\mathrm{WF} i}$, and then send them to the WFC Operator.

1) Available incremental power: For the $i$ th WF, its available incremental power $P_{\mathrm{ava}}^{\mathrm{WF} i}$ can be obtained by,

$$
P_{\mathrm{ava}}^{\mathrm{WF} i}=\sum_{j=1}^{\mathcal{N}_{\mathrm{T}}}\left(P_{\max , j}^{\mathrm{WF} i}-P_{e 0, j}^{\mathrm{WF} i}\right), \quad \forall i \in \mathcal{N}_{\mathrm{F}}
$$

where $\mathcal{N}_{\mathrm{T}}$ is the number of WTs in this WF, $P_{\max , j}^{\mathrm{WF} i}$ and $P_{e 0, j}^{\mathrm{WF} i}$ are the maximum available active power output and the measured active power output (before the disturbance) of the $j$ th WT, respectively.

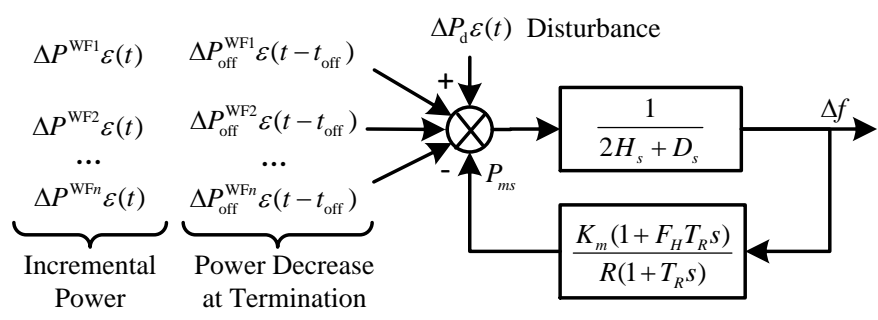

Fig. 4. low-order SFR model.

2) Relation between $\Delta P_{\mathrm{off}}^{\mathrm{WF} i}$ and $\Delta P_{\mathrm{ref}}^{\mathrm{WF} i}$ : For the $j$ th WT in the $i$ th WF, the relation between power decrease at termination $\Delta P_{\mathrm{off}, j}^{\mathrm{WF} i}$ and $\Delta P_{\mathrm{ref}, j}^{\mathrm{WF} i}$ can be approximated as,

$$
\Delta P_{\mathrm{off}, j}^{\mathrm{WF} i}=k_{j}^{\mathrm{WF} i} \Delta P_{\mathrm{ref}, j}^{\mathrm{WF} i}, \quad \forall j \in \mathcal{N}_{\mathrm{T}}
$$

where $k_{j}^{\mathrm{WF} i}$ is the approximation coefficient, and it is derived as [15],

$$
k_{j}^{\mathrm{WF} i}=\frac{-3 k_{\mathrm{MPPT}, j}^{\mathrm{WF} i} \omega_{\mathrm{r} 0, j}^{\mathrm{WF} i} t_{\mathrm{off}}}{2 H_{\mathrm{w}, j}^{\mathrm{WF} i}}, \quad \forall j \in \mathcal{N}_{\mathrm{T}}
$$

where $k_{\mathrm{MPPT}, j}^{\mathrm{WF} i}$ is the coefficient of the MPPT function and is calculated by mechanical properties of the WT, $\omega_{\mathrm{r} 0, j}^{\mathrm{WF} i}, P_{\mathrm{e} 0, j}^{\mathrm{WF} i}$ are the initial rotor speed and the initial active power output of the WT before the inertial control, respectively, and $H_{\mathrm{w}, j}^{\mathrm{WF}} i$ is the inertia constant of the WT.

Therefore, for the $i$ th $\mathrm{WF}$, the relation between the total incremental power $\Delta P_{\mathrm{ref} i}^{\mathrm{WF} i}$ and the total power decrease $\Delta P_{\mathrm{off}}^{\mathrm{WF} i}$ at the termination time can be approximated as,

$$
\Delta P_{\mathrm{off}}^{\mathrm{WF} i}=k^{\mathrm{WF} i} \Delta P_{\mathrm{ref}}^{\mathrm{WF} i}, \quad \forall i \in \mathcal{N}_{\mathrm{F}}
$$

where,

$$
k^{\mathrm{WF} i}=\frac{1}{\mathcal{N}_{\mathrm{T}}} \sum_{j=1}^{\mathcal{N}_{\mathrm{T}}} k_{j}^{\mathrm{WF} i}, \quad \forall i \in \mathcal{N}_{\mathrm{F}}
$$

After the disturbance, the WFs send the initial RoCoF at their PCCs to the WFC Operator, and the $\Delta P_{\mathrm{d}}$ can be estimated. Meanwhile, $P_{\mathrm{ava} i}^{\mathrm{WF} i}$ and $k^{\mathrm{WF} i}$ will be calculated at each WF controller and then be sent to the WFC Operator. With $\Delta P_{\mathrm{d}}, P_{\mathrm{ava} i}^{\mathrm{WF} i}, k^{\mathrm{WF} i}$, the system frequency nadirs after the disturbance can be predicted and the incremental power of the WFs can be determined at the WFC Operator.

\section{Prediction of System Frequency Nadirs}

The system frequency response (SFR) after the disturbance can be predicted based on the low-order SFR model [29], which is shown in Fig. 4. With the SFR model, the frequency response can be expressed as a function of time and active power outputs of the WFs,

$$
\Delta f(t, \Delta P)=f_{\mathrm{b}}\left(1-\frac{R}{K_{\mathrm{m}}+D R}\left[1+\alpha e^{-\xi \Omega_{n} t \sin \left(\Omega_{n} t+\phi\right)}\right]\right) \Delta P
$$

where $\Delta P$ is the total active power output of all WFs. The detailed explanation of (9) can be found in [29]. 
As can be seen from Fig. 2, the system frequency response after the disturbance has two nadirs. The first one is caused by the disturbance, and the second one is caused by the power decrease of the WFs at the termination time. The expressions of the frequency excursions at the two nadirs are given as,

$$
\begin{gathered}
\Delta f_{\mathrm{n} 1}=\Delta f\left(t_{\mathrm{n} 1}, \Delta P_{\mathrm{d}}+\Delta P_{\mathrm{ref}}\right) \\
\Delta f_{\mathrm{n} 2}=\Delta f\left(t_{\mathrm{n} 2}, \Delta P_{\mathrm{d}}+\Delta P_{\mathrm{ref}}\right)+\Delta f\left(t_{\mathrm{n} 2}, \Delta P_{\mathrm{off}}\right)
\end{gathered}
$$

where $t_{\mathrm{n} 1}$ and $t_{\mathrm{n} 2}$ are the time instants when the two frequency nadirs appear, respectively, and their values are described mathematically as the first and second positive value among the periodic solutions of the partial differential equation $\partial \Delta f / \partial t=0$, i.e.,

$$
t_{\mathrm{n} 1, \mathrm{n} 2}=\frac{1}{\Omega_{r}} \tan ^{-1}\left(\frac{\Omega_{r} T_{R}}{\xi \Omega_{n} T_{R}-1}\right)
$$

$\Delta P_{\text {ref }}$ and $\Delta P_{\text {off }}$ are the total incremental power and total power decrease at the termination time of the WFs, respectively, and can be obtained as,

$$
\begin{gathered}
\Delta P_{\text {ref }}=\sum_{i=1}^{\mathcal{N}_{\mathrm{F}}} \Delta P_{\mathrm{ref}}^{\mathrm{WF} i} \\
\Delta P_{\mathrm{off}}=\sum_{i=1}^{\mathcal{N}_{\mathrm{F}}} \Delta P_{\mathrm{off}}^{\mathrm{WF} i}=\sum_{i=1}^{\mathcal{N}_{\mathrm{F}}}\left(k^{\mathrm{WF} i} \Delta P_{\mathrm{ref}}^{\mathrm{WF} i}\right)
\end{gathered}
$$

\section{Determination of Incremental Power of WFs}

With the estimated magnitude of the disturbance $\Delta P_{\mathrm{d}}$, and the values of $P_{\text {ava }}^{\mathrm{WF}}, k^{\mathrm{WF} i}$ gathered from the WFs, the incremental power of the WFs can be determined at the WFC Operator with the objective of minimizing the frequency excursions at the frequency nadirs. Calculating the frequency excursions at the two nadirs is given as (10) and (11). In order to minimize them, the following two LP problems should be solved firstly at the WFC Operator.

$$
\begin{aligned}
\min & \Delta f_{\mathrm{n} 1} \\
\text { s.t. } & \Delta f_{\mathrm{n} 1 \geq \Delta f_{\mathrm{n} 2}} \\
& 0 \leq \Delta P_{\mathrm{ref}}^{\mathrm{WF} i} \leq P_{\mathrm{ava} i}^{\mathrm{WF} i} \quad \forall i \in \mathcal{N}_{\mathrm{F}} \\
\min & \Delta f_{\mathrm{n} 2} \\
\text { s.t. } & \Delta f_{\mathrm{n} 2} \geq \Delta f_{\mathrm{n} 1} \\
& 0 \leq \Delta P_{\mathrm{ref}}^{\mathrm{WF} i} \leq P_{\mathrm{ava}}^{\mathrm{WF} i} \quad \forall i \in \mathcal{N}_{\mathrm{F}}
\end{aligned}
$$

Let $\Delta \boldsymbol{P}_{1}$ and $\Delta \boldsymbol{P}_{\mathbf{2}}$ as the optimal solution sets of the above two LP problems, respectively. The incremental power of the WFs $\boldsymbol{\Delta} \boldsymbol{P}_{\text {ref }}=\left\{\Delta P_{\mathrm{ref}}^{\mathrm{WF} i} \mid i \in \mathcal{N}_{\mathrm{F}}\right\}$ should be determined as,

$$
\boldsymbol{\Delta} \boldsymbol{P}_{\text {ref }}= \begin{cases}\boldsymbol{\Delta} \boldsymbol{P}_{\mathbf{1}}, & \text { if } \Delta f_{\mathrm{n} 2}\left(\boldsymbol{\Delta} \boldsymbol{P}_{\mathbf{2}}\right) \geq \Delta f_{\mathrm{n} 1}\left(\boldsymbol{\Delta} \boldsymbol{P}_{\mathbf{1}}\right) \\ \boldsymbol{\Delta} \boldsymbol{P}_{\mathbf{2}}, & \text { if } \Delta f_{\mathrm{n} 2}\left(\boldsymbol{\Delta} \boldsymbol{P}_{\mathbf{2}}\right)<\Delta f_{\mathrm{n} 1}\left(\boldsymbol{\Delta} \boldsymbol{P}_{\mathbf{1}}\right)\end{cases}
$$

The determined incremental power of the WFs will then be dispatched to each WF controller.

\section{WT/BESS LEVEL ACTIVE POWER OUTPut COORDINATION}

After receiving $\Delta P_{\mathrm{ref}}^{\mathrm{WF} i}$ from the WFC Operator, the $i$ th WF controller determines the optimal incremental power of each WT/BESS in the WF in order to minimize the overdeceleration of the WTs' rotor speed, wind energy loss and degradation cost of the BESS. It is achieved by solving an MPC-based optimization problem in a distributed manner using the ADMM algorithm. In this section, the predictive model of the WF is firstly introduced. The formulation of the MPC problem and the ADMM-based solution are presented afterwards.

\section{A. Predictive Model of WF}

It is assumed that the number of WTs of each WF is $\mathcal{N}_{\mathrm{T}}$, and each WF has a BESS to provide additional active power during the inertial control process. Therefore, the predictive model of each WF can be expressed as,

$$
\begin{aligned}
& \Delta \dot{x}=\boldsymbol{A} \Delta x+\boldsymbol{B} \Delta u+\boldsymbol{E} \\
& \Delta y=\boldsymbol{C} \Delta x
\end{aligned}
$$

with $\Delta x=\left[\Delta x_{1}, \ldots, \Delta x_{\mathcal{N}_{\mathrm{T}}}, \Delta x_{E}\right]^{\mathrm{T}}, \Delta u=$ $\left[\Delta u_{1}, \ldots, \Delta u_{\mathcal{N}_{\mathrm{T}}}, \Delta u_{E}\right]^{\mathrm{T}}, \Delta y=\left[\Delta y_{1}, \ldots, \Delta y_{\mathcal{N}_{\mathrm{T}}}, \Delta y_{E}\right]^{\mathrm{T}}$,

$$
\begin{aligned}
& \boldsymbol{A}=\operatorname{diag}\left[\boldsymbol{A}_{\mathbf{1}}, \ldots, \boldsymbol{A}_{\mathcal{N}_{\mathbf{T}}}, \boldsymbol{A}_{\mathbf{E}}\right] \\
& \boldsymbol{B}=\operatorname{diag}\left[\boldsymbol{B}_{\mathbf{1}}, \ldots, \boldsymbol{B}_{\mathcal{N}_{\mathbf{T}}}, \boldsymbol{B}_{\mathbf{E}}\right] \\
& \boldsymbol{C}=\operatorname{diag}\left[\boldsymbol{C}_{\mathbf{1}}, \ldots, \boldsymbol{C}_{\mathcal{N}_{\mathbf{T}}}, \boldsymbol{C}_{\mathbf{E}}\right] \\
& \boldsymbol{E}=\operatorname{diag}\left[\boldsymbol{E}_{\mathbf{1}}, \ldots, \boldsymbol{E}_{\mathcal{N}_{\mathbf{T}}}, \boldsymbol{E}_{\mathbf{E}}\right]
\end{aligned}
$$

The predictive model of each WT is formulated as,

$$
\begin{aligned}
\Delta \dot{x}_{j} & =\boldsymbol{A}_{j} \Delta x_{j}+\boldsymbol{B}_{j} \Delta u_{j}+\boldsymbol{E}_{j} \quad \forall j \in \mathcal{N}_{\mathrm{T}} \\
\Delta y_{j} & =\boldsymbol{C}_{\boldsymbol{j}} \Delta x_{j}
\end{aligned}
$$

with $\Delta x_{j}=\left[\Delta \omega_{\mathrm{r}, j}, \Delta P_{\mathrm{e}, j}\right]^{\mathrm{T}}, \Delta u_{j}=\left[\Delta P_{\mathrm{ref}, j}\right], \Delta y_{j}=$ $\left[\Delta \omega_{\mathrm{r}, j}, \Delta P_{\mathrm{e}, j}\right]^{\mathrm{T}}$,

$$
\begin{aligned}
& \boldsymbol{A}_{\boldsymbol{j}}=\left[\begin{array}{cc}
\frac{P_{\mathrm{e} 0, j}-P_{\mathrm{m} 0, j}}{2 H_{\mathrm{t}} \omega_{\mathrm{r} 0, j}^{2}} & \frac{1}{2 H_{\mathrm{t}} \omega_{\mathrm{r} 0, j}} \\
0 & \frac{-1}{T_{\mathrm{c}}}
\end{array}\right] \quad \boldsymbol{B}_{\boldsymbol{j}}=\left[\begin{array}{c}
0 \\
\frac{-1}{T_{\mathrm{c}}}
\end{array}\right] \\
& \boldsymbol{E}_{\boldsymbol{j}}=\left[\begin{array}{c}
\frac{P_{\mathrm{m} 0, j}-P_{\mathrm{e} 0, j}}{2 H_{\mathrm{t}} \omega_{\mathrm{r} 0, j}} \\
0
\end{array}\right] \quad \boldsymbol{C}_{\boldsymbol{j}}=\left[\begin{array}{ll}
1 & 1
\end{array}\right]
\end{aligned}
$$

where $\omega_{\mathrm{r} 0, j}, P_{\mathrm{e} 0, j}, P_{\mathrm{m} 0, j}$ are the initial rotor speed, electrical power, and mechanical power of the $j$ th WT, respectively, and $H_{\mathrm{t}}$ is the inertia constant of each WT.

The predictive model of the BESS is,

$$
\begin{aligned}
\Delta \dot{x}_{\mathrm{E}} & =\boldsymbol{A}_{\mathbf{E}} \Delta x_{\mathrm{E}}+\boldsymbol{B}_{\mathbf{E}} \Delta u_{\mathrm{E}}+\boldsymbol{E}_{\mathbf{E}} \\
\Delta \dot{y}_{\mathrm{E}} & =\boldsymbol{C}_{\mathbf{E}} \Delta x_{\mathrm{E}}
\end{aligned}
$$


with $\Delta x_{\mathrm{E}}=\left[\Delta S O C, \Delta P_{\mathrm{e}, \mathrm{ESS}}, \Delta P_{\mathrm{int}}, \Delta I_{\mathrm{D}}\right]^{\mathrm{T}}, \Delta u_{\mathrm{E}}=$ $\left[\Delta P_{\text {ref }, \mathrm{ESS}}\right], \Delta y_{\mathrm{E}}=\left[\Delta S O C, \Delta P_{\mathrm{e}, \mathrm{ESS}}\right]^{\mathrm{T}}$,

$$
\begin{aligned}
& \boldsymbol{A}_{\mathbf{E}}=\left[\begin{array}{cccc}
0 & 0 & 0 & -\frac{1}{Q_{\mathrm{c}}} \\
0 & -\frac{1}{T_{\mathrm{f}}} & 0 & \frac{U_{\mathrm{D}}}{T_{\mathrm{f}}} \\
0 & -1 & 0 & 0 \\
0 & -\frac{k_{\mathrm{p}}}{T_{\mathrm{i}}} & \frac{k_{\mathrm{i}}}{T_{\mathrm{i}}} & -\frac{1}{T_{\mathrm{i}}}
\end{array}\right] \quad \boldsymbol{B}_{\mathbf{E}}=\left[\begin{array}{c}
0 \\
0 \\
1 \\
\frac{k_{\mathrm{p}}}{T_{\mathrm{i}}}
\end{array}\right] \\
& \boldsymbol{E}_{\mathbf{E}}=\left[\begin{array}{c}
-\Delta I_{\mathrm{D}}^{0} \\
0 \\
0 \\
0
\end{array}\right] \quad \boldsymbol{C}_{\mathbf{E}}=\left[\begin{array}{llll}
1 & 0 & 0 & 0 \\
0 & 1 & 0 & 0
\end{array}\right]
\end{aligned}
$$

where $Q_{\mathrm{c}}$ is the capacity of the BESS, $U_{\mathrm{D}}$ is the DC-voltage of the BESS, and $T_{\mathrm{i}}, T_{\mathrm{f}}, k_{\mathrm{p}}$ and $k_{\mathrm{i}}$ are parameters of the active power control loop of the BESS.

Based on the continuous model of the WF (18), the discrete time state space model with sampling time $\Delta T_{\mathrm{P}}$ can be expressed as,

$$
\begin{aligned}
\Delta x(k+1) & =\boldsymbol{G} \Delta x(k)+\boldsymbol{H} u(k)+\boldsymbol{E} \\
\Delta y(k+1) & =\boldsymbol{C} \Delta x(k+1)
\end{aligned}
$$

where,

$$
\boldsymbol{G}=e^{\boldsymbol{A} \Delta T_{\mathrm{P}}}, \boldsymbol{H}=\int_{0}^{\Delta T_{\mathrm{P}}} e^{\boldsymbol{A} \tau} \boldsymbol{B} \mathrm{d} \tau
$$

\section{B. MPC Problem Formulation}

For each WF, the control objectives are: 1) to improve the minimum rotor speed of all WTs, 2) to minimize the loss of wind energy, and 3) to minimize the degradation cost caused by charging and discharging of the BESS during the inertial control process. The cost functions are presented as follows:

1) Improve the minimum rotor speed. The difference of the rotor speeds among the WTs is minimized, meaning that all WTs during the inertial control converge to the same rotor speed. The cost function of the first objective can be described by,

$$
\sum_{j=1}^{\mathcal{N}_{\mathrm{T}}} \sum_{k=1}^{N_{\mathrm{p}}}\left\|\Delta \omega_{\mathrm{r}, j}(k)-\Delta \omega_{\mathrm{r}, \text { ave }}(k)\right\|^{2}
$$

where $\Delta \omega_{\text {r,ave }}$ is the average rotor speed of all WTs.

2) Minimize the energy loss due to the inertial control. The loss of captured wind energy of the $j$ th WT caused by the deceleration of the WT's rotor is denoted as $E_{\mathrm{loss}, j}$ and it can be approximated as [27],

$$
\begin{aligned}
E_{\mathrm{loss}, j} & =\left.\frac{1}{4} \rho \pi R^{2} v_{\mathrm{w}, j}^{3} \frac{R}{v_{\mathrm{w}, j}} \frac{\partial C_{\mathrm{p}, j}}{\partial \lambda_{j}}\right|_{t=0} T_{\mathrm{c}} \Delta \omega_{\mathrm{r}, j} \\
& +\left.\frac{1}{12} \rho \pi R^{2} v_{\mathrm{w}, j}^{3}\left(\frac{R}{v_{\mathrm{w}, j}}\right)^{2} \frac{\partial^{2} C_{\mathrm{p}, j}}{\partial \lambda_{j}^{2}}\right|_{t=0} T_{\mathrm{c}} \Delta \omega_{\mathrm{r}, j}^{2}
\end{aligned}
$$

Therefore, the objective of minimizing $E_{\mathrm{loss}, j}$ of the WTs is described by,

$$
\sum_{j=1}^{\mathcal{N}_{\mathrm{T}}} \sum_{k=1}^{N_{\mathrm{p}}} E_{\mathrm{loss}, j}(k)
$$

3) Minimize the degradation cost of BESS. According to [30], the ESS degradation cost can be expressed as,

$$
B C=\frac{\left(P_{\mathrm{ESS}}^{\mathrm{ch}}+P_{\mathrm{ESS}}^{\mathrm{dis}}\right) \mu_{\mathrm{ESS}} \gamma_{\mathrm{ESS}} T_{\mathrm{c}}}{a_{1}\left[a_{2}\left(1-S O C^{0}\right)+a_{3}\right] e^{a_{4} T_{\mathrm{ESS}}} E_{\mathrm{R}}\left(1-S O C^{\mathrm{ref}}\right)}
$$

The parameters in (29) and their typical values can be found in [30]. Therefore, the objective of minimizing the battery degradation of the BESS can be described by,

$$
\min \sum_{k=1}^{N_{\mathrm{p}}}\|B C(k)\|^{2}
$$

Accordingly, the overall cost function is expressed as,

$$
\begin{aligned}
& \min \sum_{j=1}^{\mathcal{N}_{\mathrm{T}}} \sum_{k=1}^{N_{\mathrm{p}}}\left\|\Delta \omega_{\mathrm{r}, j}(k)-\Delta \omega_{\mathrm{r}, \text { ave }}(k)\right\|^{2}+E_{\mathrm{loss}, j}(k) \\
& \quad+\sum_{k=1}^{N_{\mathrm{p}}}\|B C(k)\|^{2}
\end{aligned}
$$

During the control process, the active power output of each WT is constrained by the WT generator's capacity, and the BESS's output power should not exceed the charge/discharge power limit, i.e.,

$$
\begin{array}{r}
0 \leq P_{\mathrm{e} 0, j}+\Delta P_{\mathrm{ref}, j} \leq P_{\max , j}, \quad \forall j \in \mathcal{N}_{\mathrm{T}} \\
-P_{\text {dischage,max }}^{\mathrm{ESS}}<P_{\mathrm{e}, \mathrm{ESS}}+\Delta P_{\text {ref,ESS }}<P_{\text {charge,max }}^{\mathrm{ESS}}
\end{array}
$$

where $P_{\max , i}$ is the WT generator's capacity, $P_{\text {charge,max }}^{\mathrm{ESS}}$ and $P_{\text {dischage,max }}^{\mathrm{ESS}}$ are the charge and discharge power limits of the BESS, respectively.

The total incremental active power reference of the WF $\Delta P_{\text {ref }}$ is required to track the reference from the WFC Operator, such that,

$$
\sum_{j=1}^{\mathcal{N}_{\mathrm{T}}} \Delta P_{\text {ref }, j}+\Delta P_{\text {ref }, \text { ESS }}=\Delta P_{\text {ref }}^{\mathrm{WF} i}
$$

The formulated MPC problem (31)-(33) can be transformed into a standard quadratic-programming $(\mathrm{QP})$ problem. It will be solved based on ADMM algorithm.

\section{ADMM-based Solution Method}

The above MPC problem can be solved at the WF controller using centralized optimization algorithms. However, the number of WTs in a WF could be large, and the WF controller may suffer a heavy computational burden. To address this issue, an ADMM-based solution method is proposed to solve the MPC problem in a distributed manner. As such, the computational burden can be shared partly by the WTs and the BESS. ADMM is an algorithm that firstly decomposes the global optimization problem into several local sub-problems and then obtains the solution of the global problem by coordinating the solutions of the sub-problems. In other words, the ADMM can solve the global optimization problem locally in parallel, thus it can significantly improve the computation efficiency for solving a large-scale optimization problem. For the above MPC problem, the equality constraint (33) is satisfied by the 
TABLE I

STEPS OF ADMM-BASED SOLUTION METHOD

Step 1 Initialize: Assign 0 to all local, global, and dual variables on the first iteration, i.e.,

$x^{[1]}=0, z^{[1]}=0, y^{[1]}=0$

Step 2 Update $z$ : The WF controller minimizes the augmented Lagrangian with global variable $z$ as,

$$
\begin{gathered}
z^{[r+1]}=\underset{z}{\arg \min } y^{\mathrm{T}[r]}\left(x^{[r]}-z^{[r+1]}\right) \\
+\frac{\rho^{[r]}}{2}\left\|\left(x^{[r]}-z^{[r+1]}\right)\right\|_{2}^{2} \\
\text { s.t. } \boldsymbol{A} z=\boldsymbol{b}
\end{gathered}
$$

Step 3 Update $x$ : After updating $z$, the WF controller sends the $z_{j}$ to the $j$ th WT and $z_{\mathrm{BESS}}$ to the BESS. Then the WTs/BESS solve the sub-problems individually in parallel. The augmented Lagrangian of $j$ th WT is,

$$
\begin{aligned}
\mathcal{L}_{j}^{\mathrm{ADMM}} & =\Phi_{j}\left(x_{j}\right)+I_{\mathcal{X}_{j}}\left(x_{j}\right)+y_{j}^{\mathrm{T}[r]}\left(x_{j}-z_{j}^{[r+1]}\right) \\
& +\frac{\rho^{[r]}}{2}\left\|x_{j}-z_{j}^{[r+1]}\right\|_{2}^{2}
\end{aligned}
$$

$x_{j}$ is updated in each WT/BESS by minimizing $\mathcal{L}_{j}^{\mathrm{ADMM}}$, then the $x_{j}^{[r+1]}$ can be obtained by,

$x_{j}^{[r+1]}=\min \left\{\max \left\{\boldsymbol{H}_{j}+\rho^{[r]} \boldsymbol{I}^{-1}\left(\boldsymbol{g}_{j}+y_{i}^{[r]}\right.\right.\right.$

$$
\left.\left.\left.-\rho^{[r]} z^{[r+1]}\right), \underline{x_{j}}\right\}, \overline{x_{j}}\right\}
$$

Step 4 Update $y$ : For each WT/BESS, with the updated values of $x_{j}^{[r+1]}$ and $z_{j}^{[r+1]}$, the $y_{j}^{[r+1]}$ can be updated by,

$y_{j}^{[r+1]}=y_{j}^{[r+1]}+\rho\left(x_{j}^{[r+1]}-z_{j}^{[r+1]}\right)$

Step 5 Convergence check: Define the primal residuals as $h^{[r]}=$ $x^{[r]}-z^{[r]}$ and the dual residuals as $j^{[r]}=\left(z^{[r]}-z^{[r-1]}\right)$, then the iteration will be stopped when

$\left\|h^{[r]}\right\|^{2} \leq \epsilon^{\text {pri }} \cap\left\|j^{[r]}\right\|^{2} \leq \epsilon^{\text {dual }}$, where $\epsilon^{\text {pri }}$ and $\epsilon^{\text {dual }}$ are constants for the feasibility tolerances of the primal and dual feasibility conditions, respectively.

WF controller and the decomposed optimization problems and their corresponding inequality constraints are solved in parallel in WT/BESS controllers.

To implement the ADMM, the MPC-based optimization problem can be rewritten in an ADMM form as,

$$
\begin{gathered}
\min _{x} \Phi(x)+I_{\mathcal{X}}(x) \\
\text { s.t. } x-z=0 \\
x \in \mathcal{X} \\
\\
\boldsymbol{A} z=\boldsymbol{b}
\end{gathered}
$$

where $x$ is the vector of local variables, $z$ is the vector of global variables, and $I_{\mathcal{X}}(\cdot)$ is the indicator function of the inequality constraints. The argumented Lagrangian can be expressed as,

$$
\mathcal{L}^{\mathrm{ADMM}}=\Phi(x)+I_{\mathcal{X}}(x)+y^{\mathrm{T}}(x-z)+\frac{\rho}{2}\|x-z\|_{2}^{2}
$$

where $y$ is the dual variables vector, and $\rho>0$ is the augmented Lagrangian parameter. The steps of the ADMMbased solution method are listed in Table I.

\section{CASe Study}

The proposed HIC scheme is tested on 5 WFs (denoted as WF 1 5) that are integrated into the IEEE 39-bus system

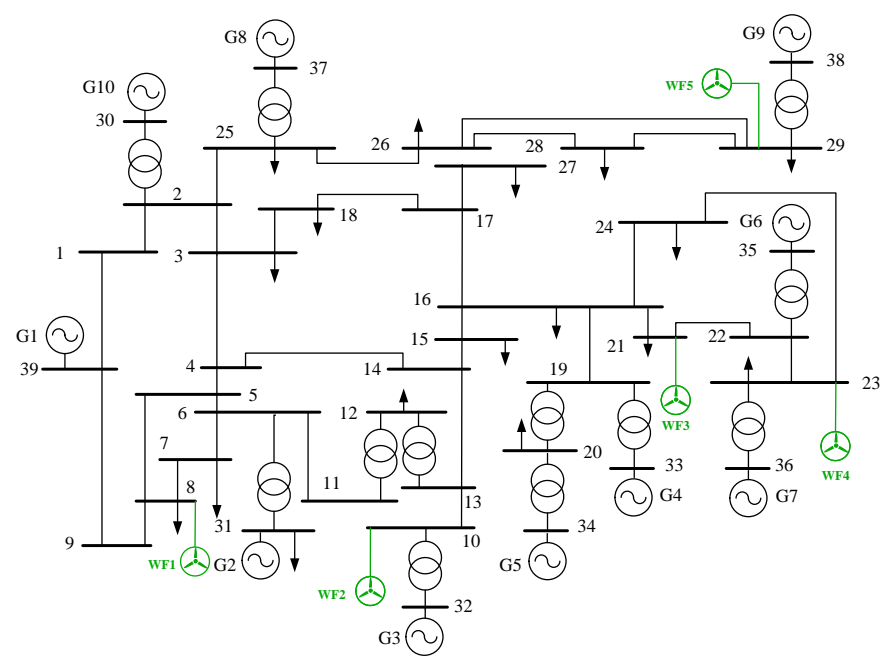

Fig. 5. Structure of the test system with 5 WFs.

TABLE II

PARAMETERS OF WT

\begin{tabular}{ccc}
\hline \hline Symbol & Description & Value \\
\hline$S$ & Rated apparent power (kVA) & 5556 \\
$P_{\mathrm{m}}^{\text {rated }}$ & Rated mechanical power (kW) & 4869.553 \\
$\omega^{\text {rated }}$ & Nominal Speed (rpm) & 1485.153 \\
$H_{\mathrm{t}}$ & Inertial constant of WT $\left(\mathrm{kg} \cdot \mathrm{m}^{2}\right)$ & 2230 \\
$H_{\mathrm{g}}$ & Inertial constant of generator $\left(\mathrm{kg} \cdot \mathrm{m}^{2}\right)$ & 170 \\
$D_{\mathrm{t}}$ & damping constant of WT & 1.5 \\
$D_{\mathrm{g}}$ & damping constant of generator & 0.1 \\
$R_{\mathrm{S}}$ & Stator Resistance (p.u.) & 0.01 \\
$X_{\mathrm{s}}$ & Stator Reactance (p.u.) & 0.1 \\
$X_{\mathrm{m}}$ & Magnetizing reactance (p.u.) & 3.5 \\
$R_{\mathrm{r}}$ & Rotor resistance (p.u.) & 0.056 \\
$X_{\mathrm{r}}$ & Rotor reactance (p.u.) & 0.031 \\
\hline
\end{tabular}

TABLE III

PARAMETERS OF BESS

\begin{tabular}{ccc}
\hline \hline Symbol & Description & Value \\
\hline$\mu_{\mathrm{ESS}}$ & Capital cost of the BESS $(\$)$ & 24000 \\
$\gamma_{\mathrm{ESS}}$ & Rated life cycle of the BESS & 1000 \\
$T_{\mathrm{ESS}}$ & Temperature of the BESS $\left({ }^{\circ} \mathrm{C}\right)$ & 25 \\
$a_{1}$ & Coefficient of battery life cycle & 3291 \\
$a_{2}$ & Coefficient of battery life cycle & -4320 \\
$a_{3}$ & Coefficient of battery life cycle & 4332 \\
$a_{4}$ & Coefficient of battery life cycle & -0.05922 \\
$E_{R}$ & Capacity of the BESS $(\mathrm{kWh})$ & 5000000 \\
$S O C^{0}$ & Initial SOC level & 0.7 \\
$S O C^{\text {ref }}$ & Reference SOC level & 1 \\
\hline \hline
\end{tabular}

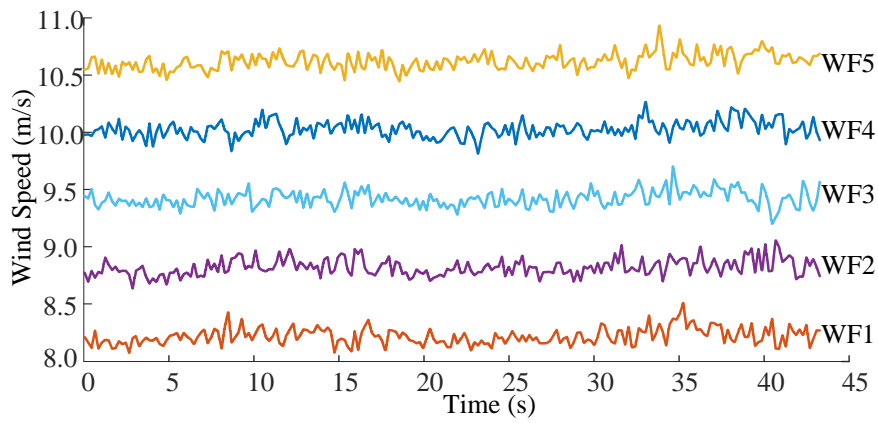

Fig. 6. Average wind speed of the WTs in each WF. 
using DIgSILENT PowerFactory. The structure of the test system is illustrated in Fig. 5. The WFs 1 5 are integrated into the system at buses $8,10,21,23,29$, respectively. Each WF has $50 \mathrm{WTs}$ and a BESS. The WTs in each WF are divided into 5 groups and each group has a different wind speed profile which is generated using the four-component wind model [31] with a different base wind speed value. The average wind speed of the WTs in each WF is shown in Fig. 6. Each WT has a capacity of $5 \mathrm{MW}$, and the BESS has a capacity of 50 MW. Thus each WF has a total capacity of 300 MW. The parameters of WTs and BESSs are listed in Tables II and III, respectively. The HIC scheme is implemented as a MATLAB script and it is connected to the PowerFactory through a cosimulation framework.

At $t=3 \mathrm{~s}$, the disturbance occurs in the power system, causing a frequency decline. The HIC scheme is activated at the same time to increase the active power outputs of the WFs and contribute to system frequency recovery. At $t=16 \mathrm{~s}$, the over-production stage of the HIC ends and the WTs enter the recovery stage.

To test the performance of WF level coordination of the proposed HIC, 3 inertial control schemes which are referred to as Scheme 1-3, respectively, were implemented at each WT and BESS. Then, the system frequency responses with HIC and Schemes 1-3 are compared. The 3 schemes are described as follows,

- Scheme 1 is proposed in [2] as the conventional synthetic inertial control scheme to make the WTs emulate the inertia and governor droop of a SG.

- Scheme 2 is proposed in [5] as an inertial control scheme with the control gains adaptive with the kinetic energy stored in the WT. The gains of the controller are set to be proportional to the spatially different levels of kinetic energy stored in different WTs and they are decreased with the declining kinetic energy during the inertial control process. In this way, the stable operation of the WTs can be ensured.

- Scheme 3 is proposed in [9] as a stepwise inertial control consisting of four stages. In Stage I, the active power output of the WT is increased by the incremental power for a preset time after detecting an event; In Stage II, the power output is decreased slowly to force the rotor speed to converge to a stable operating range; In Stage III and IV, the operating point of the WT is rapidly recovered to the MPPT point. This control scheme is designed particularly for WTs in a power system with high wind penetration level.

The performance of the proposed HIC scheme is affected by the wind penetration level and disturbance. Therefore, 3 cases were simulated by varying the wind penetration level and disturbance,

- Case 1: Wind penetration level is $23 \%$, Load 04 increases by $250 \mathrm{MW}$ at $t=3 \mathrm{~s}$.

- Case 2: Wind penetration level is $32 \%$, SG 06 trips at $t=3 \mathrm{~s}$, causing the generation loss of $240 \mathrm{MW}$.

- Case 3: Wind penetration level is $43 \%$, SG 08 trips at $t=3 \mathrm{~s}$, causing the generation loss of $150 \mathrm{MW}$.

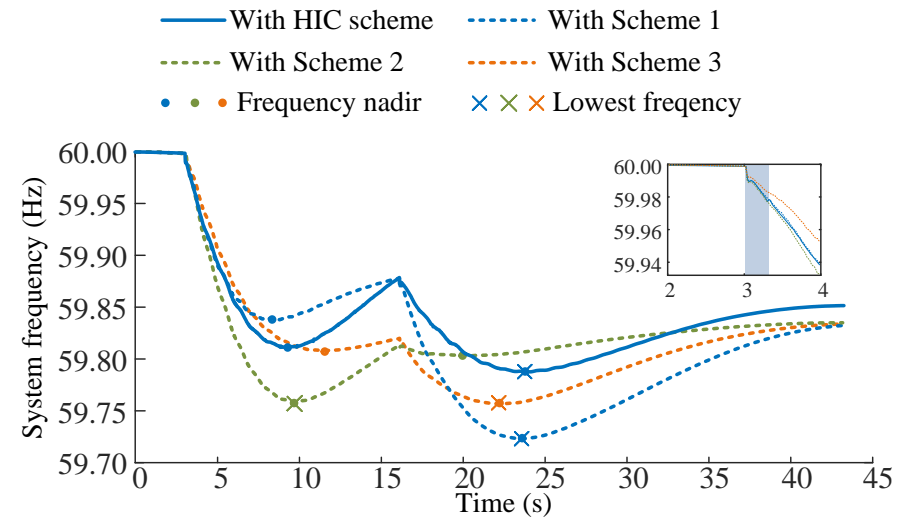

(a)

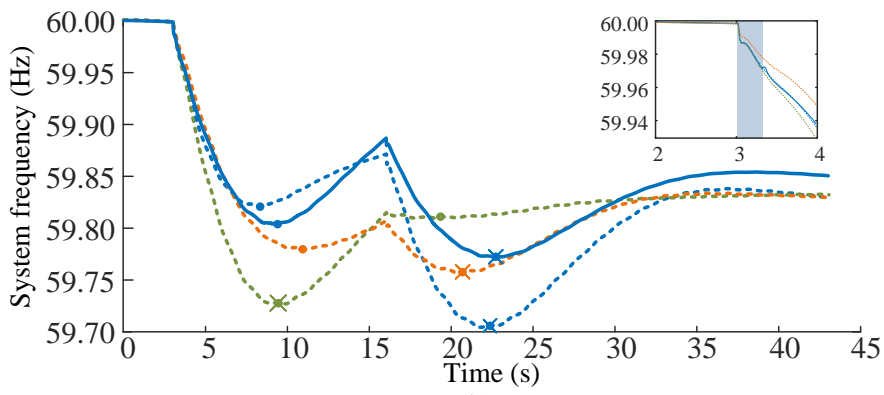

(b)

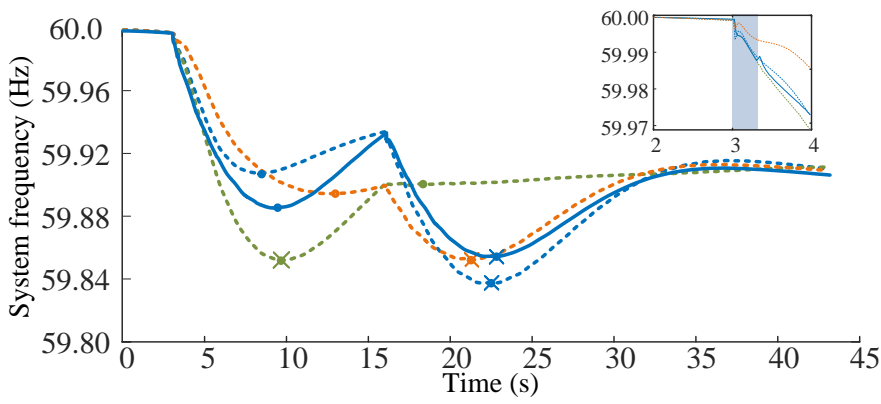

(c)

Fig. 7. System frequency response with HIC scheme and Schemes 1-3 (a) Case 1, (b) Case 2, (c) Case 3.

TABLE IV

COMPARISON OF FREQUENCY NADIRS FOR CASES 1-3

\begin{tabular}{ccccccc}
\hline \hline & \multicolumn{2}{c}{ Case 1 $(\mathrm{Hz})$} & \multicolumn{2}{c}{ Case 2 $(\mathrm{Hz})$} & \multicolumn{2}{c}{ Case 3 $(\mathrm{Hz})$} \\
\hline & Nadir 1 & Nadir 2 & Nadir 1 & Nadir 2 & Nadir 1 & Nadir 2 \\
\hline HIC & & & & & & \\
Scheme & 59.814 & 59.791 & 59.806 & 59.773 & 59.887 & 59.856 \\
Scheme 1 & 59.838 & 59.723 & 59.822 & 59.706 & 59.909 & 59.839 \\
Scheme 2 & 59.759 & 59.804 & 59.728 & 59.810 & 59.854 & 59.901 \\
Scheme 3 & 59.808 & 59.757 & 59.781 & 59.758 & 59.896 & 59.854 \\
\hline \hline
\end{tabular}

Besides, to test the performance of the WT/BESS level coordination of the HIC, a proportional hierarchical inertial control (PHIC) is built, in which the WF level coordination is the same as that of HIC, but the incremental power of the WTs and the BESS in a WF are allocated equally to satisfy the total incremental power requested by the WFC Operator. 


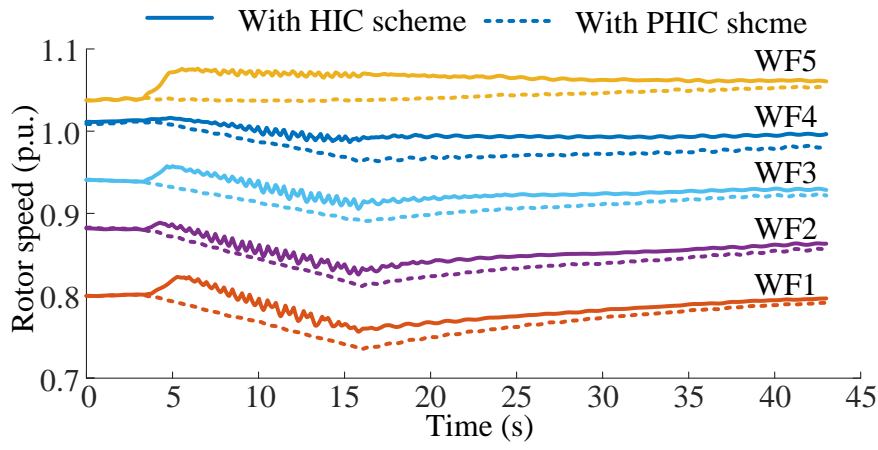

Fig. 8. Minimum rotor speed among all WTs in each WF with HIC and PHIC.

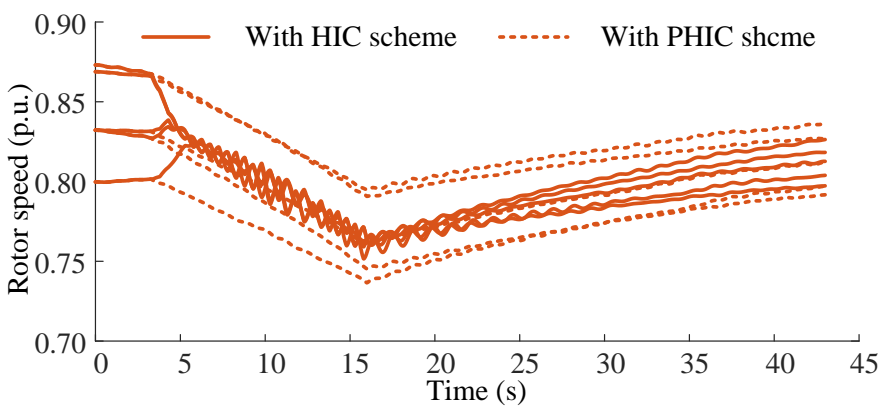

Fig. 9. Rotor speeds of WTs in WF1 with HIC and PHIC.

\section{A. Performance of WF level coordination}

The system frequency responses with the proposed HIC scheme and Schemes 1-3 in Cases 1-3 are compared in Fig. 7(a)-(c), respectively, and the system frequency excursions at the two nadirs with different schemes in Cases 1-3 are listed in Table IV. As the results show, the secondary frequency drop with Scheme 1 is severe since too much kinetic energy is released during the over-production stage of the inertial control process, causing a significant power decrease at the termination time. Scheme 2 is designed with gains decreasing with the declining kinetic energy of the WT, thus the active power output of the WT during over-production is reduced. Accordingly, the secondary frequency drop with Scheme 2 can be significantly mitigated compared with Scheme 1 . However, on the other hand, the frequency excursion at Nadir 1 becomes severer. Scheme 3 can improve both nadirs compared with Scheme 1 and 2. However, the two nadirs are still not minimized, especially in Cases 1 and 2 when the wind penetration level is low. With the proposed HIC, the lowest frequency is improved in each case compared with Schemes 1-3. The reason is that the WF level coordination is designed with the prediction of system frequency nadirs, and then the active power outputs of the WFs are determined with the objective of minimizing the two nadirs. Accordingly, the lowest system frequency can be improved, enhancing the frequency stability of the power system.

\section{B. Performance of WT/BESS level coordination}

The rotor speeds of the WTs, wind energy losses and degradation costs of the WFs with the HIC and PHIC are

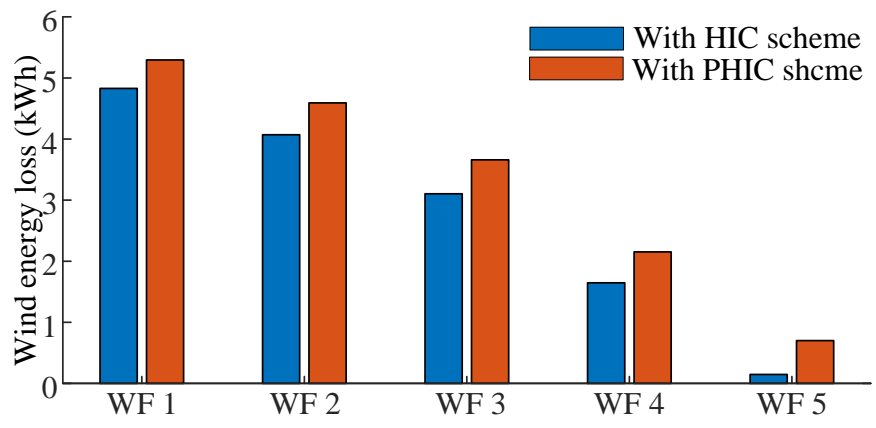

Fig. 10. Wind energy loss with HIC and PHIC.

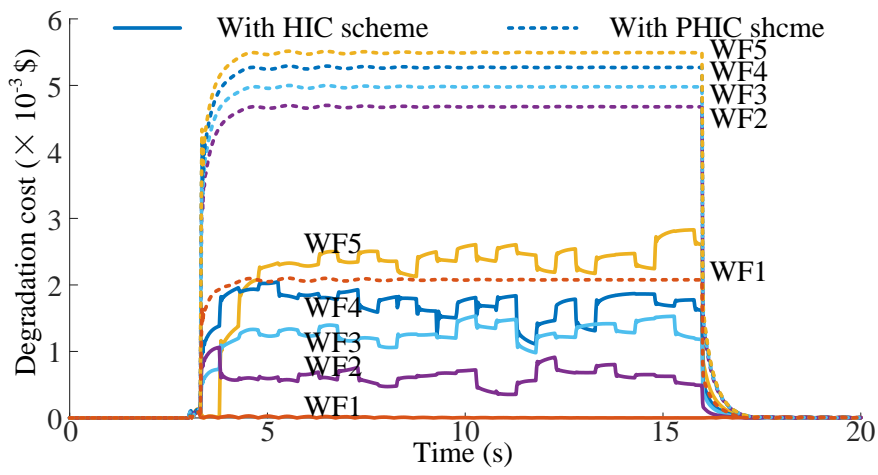

Fig. 11. Degradation cost of the BESS of each WF with HIC and PHIC.

compared for Case 1. Fig. 8 shows the minimum rotor speed among all the WTs in each WF. It can be seen that with the $\mathrm{HIC}$, the minimum rotor speed in each WF is significantly improved. To show the advantage of the HIC more clearly, the rotor speeds of all WTs in WF1 are compared in Fig. 9. Since the WTs are separated into 5 groups with different wind speed profiles, the rotor speeds of all WTs with each control scheme are presented as 5 different curves. Before the disturbance, the rotor speeds of the two control schemes are the same and all WTs operate at the MPPT point. After the disturbance, since the kinetic energy of the rotors of all WTs is released equally to the power system, the rotor speeds with the PHIC decrease with a similar ramp. Since the HIC is designed with the objective of minimizing the over-deceleration of the WTs' rotor speed, different references of active power output for the WTs are generated at the WF controller. The WTs with higher initial rotor speed have more kinetic energy, and they release more kinetic energy to the power system. Therefore, their rotor speed decreases faster than the WTs with less kinetic energy. At the end of the over-production stage, the minimum rotor speed with the HIC is 0.756 p.u., while the minimum rotor speed with the PHIC is 0.735 p.u.. Therefore, the HIC can significantly reduce the over-deceleration of the WTs' rotor speed.

The wind energy loss and degradation cost of the BESS with the HIC and PHIC are compared in Figs. 10 and 11, respectively. It can be seen from Fig. 10 that, with the HIC scheme, the wind energy loss can be significantly reduced (with an average percentage of 27.56\%) in each WF. Moreover, as Fig. 11 shows, the degradation cost of the BESS of each WF 
is also significantly reduced with the HIC. The degradation cost is associated with the charge/discharge power of the BESS, thus it is varying in each control period with the HIC since the active power output of the BESS is periodically calculated at the WF controller and then updated accordingly. Different from the HIC, the degradation cost of the BESS with the PHIC in each control period is constant since the active power output of the BESS is allocated by the WF controller as a constant. In summary, with HIC, the wind energy loss of the WTs and degradation cost of the BESS in each WF can be reduced due to the coordination of active power outputs of them. Thus, the operation cost of the WF can be reduced, which is beneficial for the WF when supporting the system frequency recovery.

\section{CONCLUSION}

This paper proposes a hierarchical inertial control scheme for multiple WFs in a power system. Firstly, the active power outputs of the WFs are optimally determined aiming at minimizing system frequency excursions at the frequency nadirs. Then, the active power outputs of the WTs and BESS in each WF are coordinated to mitigate the over-deceleration of the WTs' rotor speed and reduce the operation cost of the WF, which is achieved by formulating an MPC-based problem and solving it in a distributed manner using the ADMM algorithm.

Simulation results show that with the proposed HIC scheme, the system frequency response is improved by achieving a balance between the two frequency nadirs. Furthermore, the over-deceleration of the WTs' rotor is reduced, ensuring the stable operation of the WTs and reducing the time for WTs' rotor speed recovery. Finally, simulation results also shown decreases in the wind energy losses and the degradation costs of the BESSs in the WFs with the HIC scheme. Accordingly, the operation costs of the WFs is reduced.

\section{REFERENCES}

[1] H. T. Nguyen, G. Yang, A. H. Nielsen, and P. H. Jensen: "Combination of Synchronous Condenser and Synthetic Inertia for Frequency Stability Enhancement in Low Inertia Systems", IEEE Trans. Sustain. Energy, vol. 10, no. 3, pp. 997-1005, Jul. 2019.

[2] J. Morren, S. de Haan, W. Kling, and J. Ferreira, "Wind Turbines Emulating Inertia and Supporting Primary Frequency Control," IEEE Trans. Power Syst., vol. 21, no. 1, pp. 433-434, Feb. 2006.

[3] I. D. Margaris, S. A. Papathanassiou, N. D. Hatziargyriou, A. D. Hansen, and P. Sorensen, "Frequency Control in Autonomous Power Systems with High Wind Power Penetration," IEEE Trans. Sustain. Energy, vol. 3, no. 2, pp. 189-199, 2012

[4] J. Lee, E. Muljadi, P. Sørensen, and Y. C. Kang, "Releasable kinetic energy-based inertial control of a DFIG wind power plant," IEEE Trans. Sustain. Energy, vol. 7, no. 1, pp. 279-288, 2016.

[5] J. Lee, G. Jang, E. Muljadi, F. Blaabjerg, Z. Chen, and Y. Cheol Kang, "Stable Short-Term Frequency Support Using Adaptive Gains for a DFIGBased Wind Power Plant," IEEE Trans. Energy Convers., vol. 31, no. 3, pp. 1068-1179, Sep 2016.

[6] M. Hwang, E. Muljadi, J. W. Park, P. Sorensen, and Y. C. Kang, "Dynamic Droop-Based Inertial Control of a Doubly-Fed Induction Generator," IEEE Trans. Sustain. Energy, vol. 7, no. 3, pp. 924-933, 2016.

[7] M. Hwang, E. Muljadi, G. Jang, and Y. C. Kang, "Disturbance-Adaptive Short-Term Frequency Support of a DFIG Associated with the Variable Gain Based on the ROCOF and Rotor Speed," IEEE Trans. Power Syst., vol. 32, no. 3, pp. 1873-1881, 2017

[8] M. Kang, K. Kim, E. Muljadi, J. W. Park, and Y. C. Kang, "Frequency Control Support of a Doubly-Fed Induction Generator Based on the Torque Limit," IEEE Trans. Power Syst., vol. 31, no. 6, pp. 4575-4583, 2016.
[9] D. Yang, J. Kim, Y. C. Kang, E. Muljadi, N. Zhang, J. Hong, S. H. Song, and T. Zheng, "Temporary Frequency Support of a DFIG for High Wind Power Penetration," IEEE Trans. Power Syst., vol. 33, no. 3, pp. 3428-3437, 2018.

[10] Z. Wu, D. W. Gao, H. Zhang, S. Yan, and X. Wang, "Coordinated Control Strategy of Battery Energy Storage System and PMSG-WTG to Enhance System Frequency Regulation Capability," IEEE Trans. Sustain. Energy, vol. 8, no. 3, pp. 1330-1343, Jul. 2017.

[11] W. Bao, L. Ding, S. Yin, K. Wang, and V. Terzija, "Active rotor speed protection for DFIG synthetic inertia control," in MedPower 2016, Belgrade, Nov. 2016.

[12] F. Liu, Z. Liu, S. Mei, W. Wei, and Y. Yao, "ESO-Based Inertia Emulation and Rotor Speed Recovery Control for DFIGs," IEEE Trans. Energy Convers., vol. 32, no. 3, pp. 1209-1219, Sep. 2017.

[13] F. Hafiz and A. Abdennour, "Optimal use of kinetic energy for the inertial support from variable speed wind turbines," Renew. Energy, vol. 80, pp. 629-643, Aug. 2015.

[14] Y. Zhang, M. E. Raoufat, K. Tomsovic, and S. M. Djouadi, "Set TheoryBased Safety Supervisory Control for Wind Turbines to Ensure Adequate Frequency Response," IEEE Trans. Power Syst., vol. 34, no. 1, pp. 680692, Jan. 2019

[15] M. Kheshti, L. Ding, W. Bao, M. Yin, Q. Wu, and V. Terzija, "Toward Intelligent Inertial Frequency Participation of Wind Farms for the Grid Frequency Control," IEEE Trans. Ind. Informat., in press.

[16] Y. Li, Z. Xu, J. Zhang, and K. P. Wong, "Variable gain control scheme of DFIG-based wind farm for over-frequency support," Renew. Energy, vol. 120, pp. 379-391, May 2018.

[17] X. Tian, W. Wang, Y. Chi, Y. Li, and C. Liu, "Virtual inertia optimisation control of DFIG and assessment of equivalent inertia time constant of power grid," IET Renew. Power Gener., vol. 12, no. 15, pp. 1733-1740, Nov. 2018

[18] Z. Chen, H. Wang, and Q. Jiang, "Optimal control method for wind farm to support temporary primary frequency control with minimised wind energy cost," IET Renew. Power Gener, vol. 9, no. 4, pp. 350-359, May 2015.

[19] A. De Paola, D. Angeli, and G. Strbac, "Scheduling of Wind Farms for Optimal Frequency Response and Energy Recovery," IEEE Trans. Control Syst. Technol., vol. 24, no. 5, pp. 1764-1778, Sep. 2016.

[20] H. Zhao, Q. Wu, S. Hu, H. Xu, and C. N. Rasmussen, "Review of energy storage system for wind power integration support," Appl. Energy, vol. 137, pp. 545-553, Jan. 2015.

[21] B. Peng, F. Zhang, J. Liang, L. Ding, Z. Liang, and Q. Wu, "Coordinated control strategy for the short-term frequency response of a DFIG-ES system based on wind speed zone classification and fuzzy logic control," Int. J. Elec. Power \& Energy Syst., vol. 107, pp. 363-378, May 2019.

[22] L. Miao, J. Wen, H. Xie, C. Yue, and W. J. Lee, "Coordinated Control Strategy of Wind Turbine Generator and Energy Storage Equipment for Frequency Support," IEEE Trans. Ind Appl., vol. 51, no. 4, pp. 27322742, Jul.-Aug. 2015.

[23] M. Rezkalla, M. Pertl, and M. Marinelli, "Electric power system inertia: requirements, challenges and solutions," Electrical Engineering, vol. 100, no. 4 , pp. 2677-2693, Dec. 2018.

[24] X. Bai, J. Wang, X. Wan, M. Peng, X. Zhang, and J. Geng, "Effects of Large-Scale Wind Integration on China Northwest Regional Power System and Future Solutions, in the 18th IFAC World Congress, Milano, 2011.

[25] F. Baccino, F. Conte, S. Grillo, S. Massucco, and F. Silvestro, "An Optimal Model-Based Control Technique to Improve Wind Farm Participation to Frequency Regulation," IEEE Trans. Sustain. Energy, vol. 6, no. 3, pp. 993-1003, Jul. 2015.

[26] Z. Wang and W. Wu, "Coordinated Control Method for DFIG-Based Wind Farm to Provide Primary Frequency Regulation Service," IEEE Trans. Power Syst., vol. 33, no. 3, pp. 2644-2659, May 2018.

[27] S. Wang and K. Tomsovic, "A Novel Active Power Control Framework for Wind Turbine Generators to Improve Frequency Response," IEEE Trans. Power Syst., vol. 33, no. 6, pp. 6579-6589, Nov. 2018.

[28] V. Terzija, "Adaptive underfrequency load shedding based on the magnitude of the disturbance estimation," IEEE Trans. Power Syst., vol. 21 , no. 3, pp. 1260-1266, Aug. 2006.

[29] P. Anderson and M. Mirheydar, "A low-order system frequency response model," IEEE Trans. Power Syst., vol. 5, no. 3, pp. 720-729, Aug. 1990.

[30] S. Huang, Q. Wu, Y. Guo, and F. Rong, "Hierarchical active power control of DFIG-based wind farm with distributed energy storage systems based on ADMM," IEEE Trans. Sustain. Energy, in press.

[31] P. Anderson and A. Bose, "Stability Simulation Of Wind Turbine Systems," IEEE Trans. Power App. Syst., vol. PAS-102, no. 12, pp. 37913795, Dec. 1983. 